\title{
PROBLEMÁTICA EDUCATIVA DEL ALUMNADO DE ALTAS CAPACIDADES. ANÁLISIS DE LAS PERCEPCIONES DE FAMILIAS Y EDUCADORES
}

\author{
EDUCATIONAL PROBLEMS REGARDING GIFTED STUDENTS. \\ ANALYSIS OF FAMILIES AND EDUCATORS' PERCEPTION
}

\author{
M. ${ }^{a} L$. Pontón*, S. Fernández** \\ Universidad de Oviedo
}

\section{RESUMEN}

Este trabajo analiza las percepciones que tienen las familias y los educadores sobre el desarrollo personal, familiar y cognitivo de los niños y niñas de altas capacidades.

Se estudian los aspectos coincidentes y discrepantes entre padres, madres y educadores con el fin de comprender e identificar las causas que dificultan la buena comunicación y colaboración. Se pone de manifiesto que uno de los aspectos más importantes es la relación que las familias mantienen con los centros.

Palabras claves: Altas capacidades, familia, educadores y colaboración.

\section{ABSTRACT}

This essay analyses the perceptions that families, counsellors and teachers have regarding the personal and cognitive development of gifted children.

We will study the coinciding and opposing aspects among mothers, fathers and educators with the objective of understanding and identifying the causes that obstruct good communication and collaboration. We will highlight the fact that one of the most important aspects is the relationship that the families maintain with the educational centres.

Key words: Gifted children, families, educators and collaboration.

* Licenciada en Filosofía y Ciencias de la Educación. Profesora de E.S.O. Línea principal de investigación: educación del alumnado de altas capacidades.

** Profesor de Metodología de Investigación y Diagnóstico en Educación de la Univ. de Oviedo. Línea principal de investigación: Metodología ODI de intervención educativa especializada. 


\section{Las familias y los educadores ante las altas capacidades}

La inquietud social por atender al alumnado de altas capacidades se ha concretado desde hace tiempo en diversos estudios e investigaciones, aunque ha sido en la pasada década cuando el sistema educativo español ha plasmado en su legislación (BOE 2-VI-95 y BOE 16-V-96) la acción educativa especializada para dicho alumnado. Esta tardanza en regular una acción educativa puede explicarse por los recelos y prejuicios que todavía hoy suscita la educación especializada de este alumnado.

En los últimos años se ha puesto en evidencia la necesidad de desarrollar una política educativa que diera respuesta a las demandas del alumnado con necesidades especiales, dadas sus características singulares (discapacitados, alumnado con problemas de aprendizaje y carencias de todo tipo). Consecuentemente, se han realizado diversas actuaciones y medidas educativas que tratan de evitar que las diferencias se conviertan en desigualdades y desventajas. En este sentido, la L.O.G.S.E. introdujo la atención a la diversidad como una forma de atender las diferencias individuales, y esto incluye al alumnado de altas capacidades, dado que también necesitan medidas educativas específicas. Es, pues, necesario acometer una educación que posibilite a este alumnado poder alcanzar un pleno desarrollo, acorde con sus características y necesidades concretas.

Para alcanzar ese pleno desarrollo resulta de vital importancia el papel de la familia, que debe ser la base que sustente el estímulo y el apoyo necesario. Si educar no es una tarea sencilla, parece que ésta se complica y se llena de interrogantes y dificultades cuando se afronta con niños con esas características. Averiguar qué rol juega la familia y los educadores, es el propósito del trabajo.

\section{Las familias y los hijos de altas capacidades}

Los estudios realizados en el campo de las altas capacidades no resultan abundantes y los existentes han dedicado más atención a las necesidades intelectuales y de aprendizaje, obviando la problemática emocional, personal y familiar (Domínguez y Pérez, 1998).

A pesar de esta situación, se puede apreciar un cierto interés por el estudio del entorno familiar de los niños de altas capacidades; pero, especialmente, ha sido en los años 70 y 80 cuando algunos investigadores han puesto de relieve la importancia de la familia en el desarrollo de estos niños y la necesidad de una mayor investigación en tal dirección, como fueron los casos modélicos de Hill (1980) y Tabackman (1976).

Las escasas investigaciones realizadas, están centradas sobre todo en la influencia de las familias. Bloom (1985) y Pressey (1955) enfatizan el decisivo papel de los padres y otros miembros de la familia. Así, creen que juegan un papel muy significativo en la identificación y la educación, a la vez que consideran el apoyo hacia sus hijos una pieza crucial, aunque, evidentemente, no la única.

Gottfried, A. W., Gottfried, A. E., Bathurst, y Guerin (1994) muestran cómo estos hogares suelen estar «enriquecidos» desde la infancia. De acuerdo con Bloom (1985) y Howe (1990) las altas capacidades parece que nunca se llegan a conseguir sin que al menos uno de los padres o algún familiar directo o ambos, trabajen muy duro, dirijan al hijo o a la hija 
a esforzarse, les inculquen el aprovechamiento del tiempo y la necesidad de llegar a alcanzar unas metas muy altas con una fuerte disciplina.

Silverman (1986b) sostiene que los padres perciben las altas capacidades como éxitos, mientras que las madres los entienden en términos ligados al desarrollo de las diferencias. Cornell (1983) señala que esta situación a veces provoca en las parejas una manera diferente de entender la educación; mientras que la madre prefiere facilitarle experiencias ricas y variadas, que estimulen y favorezcan su desarrollo, aunque no se coronen con altos logros, el padre, aunque es más escéptico ante el diagnóstico, tiende a exigir un mayor rendimiento.

Los trabajos publicados basados en la influencia que ejercen los hijos con altas capacidades son escasos, como así lo manifiestan Ballering y Koch (1983), pero describen muy bien los cambios que se observan en el entorno familiar.

La familia, ante el diagnóstico de sus hijos, reacciona de forma positiva, negativa e, incluso, con rechazo. Unos lo admiten como un premio, algunos como un castigo, y otros, como una forma de brillar socialmente; mientras que unos quieren que desarrollen al máximo sus potencialidades, otros dudan, porque les preocupa que eso suponga aislamiento (Domínguez y Pérez, 1998).

Sin embargo, a pesar de que ciertos autores también consideran que cada contexto familiar puede recibir de forma diferente a su hijo o hija, tienden a describir la relación como ambivalente. La superdotación genera al mismo tiempo reacciones opuestas, de aceptación y rechazo, según hacen ver Colangelo, Davis (1991) y Gallagher (1991). Así pues, parece que tener hijos de altas capacidades no implica que se produzcan siempre las situaciones anteriormente descritas, pero sí ocasiona cambios en la vida cotidiana.

La mayoría de padres y madres que participaron en la investigación de Hackney (1981), manifestaron que la vida familiar se alteraba por la presencia de un hijo o hija de altas capacidades.

Estos niños se interesan desde edades muy tempranas por asuntos complejos, lo que causa perplejidad en los progenitores. Es difícil aceptar y afrontar esas peculiaridades puesto que los grandes cambios en su forma de actuar provocan una mezcla de contradicciones. Por otro lado tienden a ser muy activos cuando son pequeños, suelen dormir poco y responden intensamente a su entorno, ocasionando agotamiento e insatisfacción a quienes les rodean.

El comportamiento social de sus hijos también puede llegar a producir mucha inquietud. Con frecuencia disfrutan jugando con otros mayores que ellos, cuidando a niños más pequeños o hablando con adultos. Sin embargo, las relaciones con los de su misma edad suelen ser conflictivas.

Miller (1991) describe la dependencia afectiva que genera la relación familiar y los problemas que acarrea, sobre todo, cuando padres y madres intentan realizarse en sus hijos. La percepción que se tiene de las altas capacidades, deformada por estereotipos, también suele producir en las familias inseguridad y miedo a verse desbordadas.

Tener niños con esas características representa una preocupación constante, puesto que realizan una interpretación un tanto catastrofista al pensar que cualquier acontecimiento puede provocar en sus hijos, tan delicados y frágiles, males irremediables. 
Son numerosos los estudios que demuestran cómo los conflictos aumentan cuando se detectan estas capacidades (Cornell, 1984 y Dirks, 1979). Así nos encontramos con hostilidad social, encubierta o manifiesta, hacia los intelectualmente dotados; con falta de apoyo social para su diagnóstico y estimulación; con carencia de información sobre recursos válidos; con falta de recursos económicos; y con un desarrollo irregular de la personalidad de estos niños (Ness, 1988). Según Martínez y Castiglione (1996), la mayoría de los estudios son limitados pues carecen de aproximaciones fundamentales bien en un marco teórico, bien en evidencias empíricas o experimentales.

Las dos preocupaciones más frecuentes, a juicio de Colangelo y Dettmann (1983), son el papel que desempeña el niño o la niña superdotado/a y las expectativas que genera. Asimismo, los problemas surgen porque las familias perciben que las necesidades de sus hijos son distintas y desconocen cómo atenderlas. Esta reacción, común en padres y madres de cualquier niño o niña discapacitado/a, no es reconocida en el caso de las altas capacidades, debido a la falta de información y a la deformación existente.

Todo esto se agrava, cuando se posee un bajo nivel cultural, ya que crece el deseo de que sus descendientes no presenten altas capacidades, puesto que no los entienden. No parece, por lo tanto, tan extraño y contradictorio que no se acepte de buen grado que sus hijos sean especiales, «raros», (Ross, 1964).

Las familias se sienten insatisfechas porque no se consideran suficientemente preparadas para apoyar y ayudar a sus hijos. Colangelo y Dettman (1980) lo describen como inadecuación. Esta inseguridad proviene de la falta de conocimiento y desencadena un estado de frustración y tensión (Bridges, 1973 y Pérez Sánchez, 1993, 1995). Moon, Kelly y Feldhusen (1977) indican que para aliviar la tensión es necesario información y orientación, especialmente aquella relacionada con los roles y la dinámica familiar

No siempre se recibe con agrado la sospecha o confirmación de que tienen un hijo o hija con estas características, pero pasado el primer temor, se suele sentir satisfacción, tal como nos indica Verhaären (1991). Esta actitud inicial de rechazo puede venir precedida de la necesidad de conocer y comprender cómo es su hijo y qué se espera de él.

Algunas familias llegan a comprender que es un miembro más, por lo que suelen pasar del temor al más íntimo orgullo. En ocasiones se acepta desde el primer momento, aunque esto es más frecuente cuando se posee confianza, como padres y educadores. En ese caso, la principal preocupación suele consistir en estimular sistemáticamente su desarrollo personal e intelectual desde edades tempranas. Este tipo de actitud predomina entre las clases acomodadas y en los lugares donde la escuela y la comunidad apoyan las necesidades educativas de este alumnado, tal y como señalan Coriat (1990) y Wallace (1988).

\section{La relación familia-centro ante las altas capacidades}

Muchos autores consideran que la relación que mantienen las familias del alumnado con altas capacidades con el colegio, es uno de los puntos más conflictivos. Coleman (1985) asegura que, cuando la escuela reconoce oficialmente que su hijo presenta altas capacidades, puede generar una preocupación en sus padres que previamente dormía o no existía. Las familias se plantean muchos interrogantes, que en la mayoría de las ocasiones no sabe contestar, y que van, desde iniciar la búsqueda de mejores oportunidades educativas para 
ellos, hasta ignorar el asunto con la esperanza de que desaparezca. Acedera (2000) destaca la confusión y el desconocimiento sobre la función a realizar y la actuación educativa más conveniente para sus hijos.

Los distintos puntos de vista entre familia y escuela, sobre todo cuando no hay un buen rendimiento, son muy habituales, tal como refleja Butler-Por (1993). George (1988), manifiesta que a los padres y a las madres se les reprochan sus demandas por considerarlas irracionales, y por su actitud hostil hacia los centros. Como vemos, se debe tratar de mejorar la relación familia-centro y para ello, resulta necesario identificar los problemas, tratarlos abiertamente y buscar soluciones.

Arocas, Martínez y Regadera (1999), sin embargo, sostienen que el nivel de satisfacción de los progenitores con respecto a la atención que reciben sus hijos en las escuelas, es positivo y que la mayoría se muestra satisfecha con la educación que reciben. Creen que hay que abandonar el estereotipo sobre una actitud hipercrítica e, incluso, negativa hacia la educación.

Martínez y Castiglione (1995 y 1996) afirman que se han consolidado estereotipos y mitos que poco tienen que ver con el conjunto de «sujetos excepcionales» y que están deformando la realidad. Esto es la causa que obstaculiza en muchas ocasiones la relación familia-centro. Los más significativos son: las altas capacidades están directamente relacionadas con el fracaso escolar; los intereses de estos niños son incompatibles con los de los demás; y la existencia de un desequilibrio entre el desarrollo emocional y cognitivo. Además, gran parte de los pequeños no son nunca reconocidos como tales por las falsas ideas preconcebidas que tienen muchos profesionales.

Respecto a los estereotipos sobre las familias, predomina una imagen negativa y distorsionada. En muchos casos se considera que su único objetivo es convertir a su hijo/a en la «estrella» que su propio ego narcisista necesita, como recuerda Cornell (1984). Les enseñan que son superiores a los demás, así como realizan comparaciones envidiosas entre sus hijos, a la vez que se jactan ante los demás de su condición, tienen expectativas irreales, son competitivos y celosos, sobreprotegen y son incompetentes en la tarea de enseñar. George (1988) asegura que se les acusa de elitistas y de pretender cambiar la estructura de la escuela en beneficio de sus hijos. Sin embargo, suelen tener buenas relaciones, presentando un nivel de ajuste psicológico menos conflictivo y más alto que la mayoría (Mathews, West y Hosie, 1986) y una gran cohesión (Robinson y Noble 1991).

Algunos investigadores afirman que, incluso entre el profesorado, existen muchos estereotipos. Se observa un gran desconocimiento, incapacidad y poca información sobre cual es la situación de este alumnado y la manera de responder a sus necesidades educativas Acedera y Sastre (1998). Respecto a la relación que mantienen con las familias, este primer autor, Acedera (2000) destaca que los docentes tienden a pensar que los padres son agresivos, egoístas y que presionan en exceso. Esto, unido a que tanto unos como otros plantean generalmente una concepción muy diferente sobre cómo se debe enseñar, genera conflictos y dificulta una buena relación.

Parece que existe un gran desconocimiento, incertidumbre y desinformación sobre la verdadera situación del alumnado de altas capacidades. Este estudio pretende conocer lo que realmente piensan familias y educadores sobre los niños de altas capacidades. Para conseguirlo se parte del pensamiento de Morton y Workman, citados en Hackney (1981), Gardner (1995), Wallace y Walberg (1987), coincidentes en que los niños de altas capacidades 
deben ser percibidos y estudiados como parte de un mayor ecosistema que incluya el mundo interior del pequeño, la familia, las condiciones sociales y el colegio.

\section{Estudio sobre la percepción de las altas capacidades por familias y educadores*}

Para tratar de conocer la visión que tienen las familias con hijos de altas capacidades y su relación con los centros educativos, se ha llevado a cabo un estudio en el que se contrastan las opiniones de éstas con las de orientadores y tutores. Este trabajo se plantea:

1. Analizar la opinión de los educadores y la familia de niños con altas capacidades en relación con la situación familiar, la política educativa y la actuación educativa en las aulas.

2. Averiguar el tipo de relación que se establece entre las familias con hijos de altas capacidades y los centros educativos.

\section{Metodología}

La variable genérica de este estudio es la percepción que tienen los padres, madres, tutores y orientadores sobre los niños de altas capacidades y su entorno (especialmente familiar). Esta variable genérica se analiza en seis niveles: la situación de los niños de altas capacidades, la situación de la familia, la política educativa, los colegios, las aulas y la colaboración familia-escuela. Asimismo, se han desglosado esos seis apartados en distintos indicadores.

\section{Instrumentación}

Ha sido necesario realizar un cuestionario de opinión para la recogida de información que ofreciera las suficientes garantías de validez y fiabilidad. En la elaboración del cuestionario, se han seguido los siguientes pasos formulados por Selltiz (1976):

\section{a) Decisión de la información a buscar}

Se obtuvo de las familias, tutores y orientadores, información relativa a la influencia que ejercen los niños de altas capacidades sobre sus progenitores, la verdadera situación familiar, los puntos coincidentes y discrepantes sobre los centros escolares, las actuaciones en las aulas y la colaboración familia-escuela.

\section{b) Decisión sobre el tipo de cuestionario a utilizar}

A partir de la información recopilada en diversas lecturas, seminarios y conferencias, así como de contactos con familias y educadores, se seleccionaron los contenidos específicos más pertinentes. Se combinaron preguntas abiertas, semicerradas y cerradas con la finalidad de recoger aspectos considerados importantes para la investiga-

\footnotetext{
* Dejamos constancia del agradecimiento a los centros, familias y profesionales que facilitaron la realización de este trabajo.
} 
ción, así como opiniones manifestadas libre y abiertamente por los entrevistados. Para responder a las preguntas cerradas se ofrecieron cinco alternativas. En los casos en que fue posible se realizó también una entrevista.

\section{c) Revisión de las preguntas, elaboración del cuestionario definitivo}

Se aplicó un primer cuestionario a colaboradores, lo que permitió cambiar algunos términos que parecían confusos, y se estructuraron algunas preguntas.

Se realizaron entrevistas personales, ya que se consideró esta fórmula mucho más enriquecedora, al poder explicar los objetivos y ofrecer una ayuda en caso de que no se comprendiera muy bien lo que se pedía.

\section{Composición de la muestra}

De una población de 47 escolares de altas capacidades, identificados en la comunidad autónoma del Principado de Asturias, han aceptado colaborar en el estudio 46 padres y madres de 27 alumnos y alumnas de altas capacidades. Entre este alumnado existe una alta proporción de varones $(77,8 \%)$. Catorce niños no realizan ningún tipo de actividad especial (programas de enriquecimiento y aceleración). Poseen, en general, un rendimiento académico bueno; tienen una edad media de 12 años; y se les diagnostican las altas capacidades a los 6 años.

De las 46 familias (26 madres y 20 padres) que participaron en el estudio, 19 pertenecen a la asociación de padres y madres de altas capacidades (APADAC), lo que representa un $41,3 \%$, no perteneciendo el resto a ninguna asociación de este tipo. La mayoría poseen estudios medios.

También se ha entrevistado a 62 profesionales de la educación; de ellos, 40 son orientadores; y el resto, docentes. El 80\% del profesorado son maestros, imparten clase predominantemente en colegios públicos $(60 \%)$, de diversos cursos, desde infantil hasta COU, aunque la mayoría están en Primaria (50\%) y en Secundaria (32\%)

La muestra total es de 108 personas entre padres, madres, tutores y orientadores.

\section{Técnicas de análisis}

Los análisis efectuados han sido de cuatro tipos:

1. Fiabilidad del cuestionario mediante el coeficiente alfa de Crombach.

2. Contraste de opiniones en forma descriptiva, utilizando pruebas no paramétricas (tests de la Mediana).

3. Reducción de las variables a factores más homogéneos por medio de un análisis factorial.

4. Análisis diferencial de los factores mediante Anovas.

Los datos han sido analizados mediante el programa SPSS. 9.0. y se ha contado con la colaboración del servicio común Consultoría Estadística de la Universidad de Oviedo. 


\section{Fiabilidad del cuestionario}

Tras una primera descripción de la muestra, se realiza un análisis de la fiabilidad del cuestionario para conocer la consistencia de los resultados. Se utiliza el coeficiente alfa de Crombach, ya que los items admiten una gradación de la respuesta correcta. También se consideró conveniente la supresión de algunas de las preguntas, dado que el número de encuestados que han respondido «efectivamente» a todas las preguntas fue escaso, se reemplazaron, tanto los datos perdidos como los valores NS/NC por la mediana muestral, al ser las distribuciones asimétricas.

La fiabilidad medida mediante el coeficiente alfa de Crombach presenta un valor de 0.7671, por lo que resulta conveniente eliminar algunos items. Como consecuencia de esta supresión, nos hemos quedamos con una batería de 45 preguntas y una fiabilidad de 0.8007 , en donde las correlaciones están más o menos compensadas.

\section{Contraste de opiniones}

Se efectúa un análisis general de la encuesta, pregunta a pregunta, asociando algunas cuestiones propuestas (excluyendo también aquí los items eliminados en el análisis de la fiabilidad). Se comprueba en las variables resultantes las posibles diferencias en función de la «relación» que se tiene con el alumnado considerado en este trabajo; para ello se realizan pruebas no paramétricas (test de la Mediana).

Para crear las variables de agrupación, se calcula la media entre las puntuaciones obtenidas en las preguntas pertenecientes a cada grupo; no se ha tenido en cuenta a los individuos que en alguna de las cuestiones pertenecientes a cada grupo tengan un NS/NC (no se efectuará el reemplazamiento anterior).

Los resultados más importantes son:

- PROBLEMÁTICA EMOCIONAL, SOCIAL Y ESCOLAR. Este bloque de información se obtuvo a partir de las variables reflejadas en el siguiente cuadro:

\begin{tabular}{|c|}
\hline \multicolumn{1}{|c|}{ Variables agrupadas } \\
\hline $\begin{array}{l}\text { 1- Problemas de madurez y } \\
\text { desarrollo emocional. }\end{array}$ \\
\hline 2- Problemas de interacción social \\
\hline 3- Problemas escolares \\
\hline
\end{tabular}

\begin{tabular}{|c|c|c|c|c|c|c|c|}
\hline \multicolumn{8}{|c|}{ Problemática escolar, social y emocional } \\
\hline & & Media & d.t. & Mín. & $\mathbf{P}(\mathbf{5 0})$ & Máx. & $\mathbf{N}$ \\
\hline \multirow{3}{*}{ Relación } & $\mathrm{M} / \mathrm{P}$ & 1,65 & 0,51 & 1,00 & 1,67 & 3,00 & 45 \\
\hline & $\mathrm{O}$ & 1,86 & 0,44 & 1,00 & 2,00 & 2,67 & 39 \\
\hline & $\mathrm{T}$ & 2,03 & 0,67 & 1,00 & 2,00 & 3,33 & 22 \\
\hline \multicolumn{2}{|c|}{ Total } & 1,81 & 0,54 & 1,00 & 1,67 & 3,33 & 106 \\
\hline
\end{tabular}

Los resultados han sido valorados en una escala entre 1 y 4 en los diferentes items. A la respuesta «muy de acuerdo» se le ha dado un valor aleatorio de uno, a la respuesta «de acuerdo», un dos, al «desacuerdo», tres y al «muy en desacuerdo» un valor de cuatro. Los estadísticos descriptivos utilizados en las tablas son los siguientes: $\mathrm{M} / \mathrm{P}$ se refieren a madres y padres, $\mathrm{O}$ a orientadores y $\mathrm{T}$ a tutores, la desviación típi- 
ca (d.t.), el valor mínimo y máximo (Min. y Máx.), la mediana $\mathrm{P}(50)$ y por último $\mathrm{N}$ que se refiere al número de personas que han contestado.

Se agrupan los items relacionados con el aspecto estudiado (en este caso la problemática) y se calcula la media.

Se aprecian diferencias $(p=0.021)$ en las respuestas de los encuestados, estando las familias más de acuerdo con la existencia de una problemática emocional, social y escolar, más moderadamente los orientadores y más dispersas las opiniones de los tutores.

- GÉNERO. En este módulo se recoge la información de las cuestiones referidas al género:

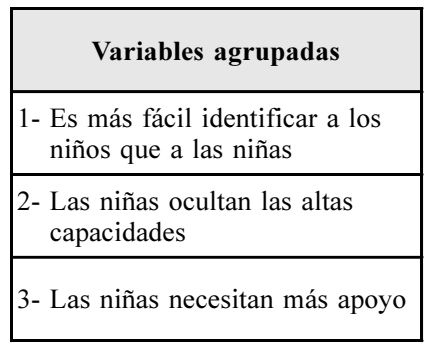

\begin{tabular}{|c|c|c|c|c|c|c|c|}
\hline \multicolumn{8}{|c|}{ Género } \\
\hline & & Media & d.t. & mín & $P(50)$ & máx & $\mathbf{N}$ \\
\hline \multirow{3}{*}{ Relación } & $\mathrm{P} / \mathrm{M}$ & 2,24 & 0,77 & 1,00 & 2,00 & 3,67 & 26 \\
\hline & $\mathrm{O}$ & 2,69 & 0,82 & 1,00 & 3,00 & 4,00 & 32 \\
\hline & $\mathrm{T}$ & 2,92 & 0,24 & 2,33 & 3,00 & 3,00 & 8 \\
\hline \multicolumn{2}{|c|}{ Totales } & 2,54 & 0,79 & 1,00 & 2,83 & 4,00 & 66 \\
\hline
\end{tabular}

En función del tipo de relación, aunque siempre muy sensiblemente $(\mathrm{p}=0.012)$, se observa que las familias están más de acuerdo con las tres propuestas relativas al género (las niñas se identifican peor porque ocultan las altas capacidades y necesitan más apoyo), mientras que los tutores están más en desacuerdo. Los porcentajes de desconocimiento son muy altos, llegando en los tutores a un valor superior al $50 \%$.

- EDUCACIÓN DE HIJOS E HIJAS. Se busca información mediante las variables del cuadro:

\begin{tabular}{|c|}
\hline \multicolumn{1}{|c|}{ Variables agrupadas } \\
\hline $\begin{array}{l}\text { 1- Complejidad en educar a estos } \\
\text { niños }\end{array}$ \\
\hline $\begin{array}{l}\text { 2- Falta de preparación de las } \\
\text { familias }\end{array}$ \\
\hline $\begin{array}{l}\text { 3- Necesidad de orientación y } \\
\text { formación }\end{array}$ \\
\hline
\end{tabular}

\begin{tabular}{|c|c|c|c|c|c|c|c|}
\hline \multicolumn{7}{|c|}{ Educación de hijos/as } \\
\hline & & Media & d.t. & mín & P(50) & Máx & N \\
\hline \multirow{3}{*}{ Relación } & $\mathrm{P} / \mathrm{M}$ & 1,77 & 0,58 & 1,00 & 1,67 & 3,00 & 44 \\
\cline { 2 - 8 } & $\mathrm{O}$ & 1,79 & 0,47 & 1,00 & 1,67 & 3,00 & 38 \\
\cline { 2 - 8 } & $\mathrm{T}$ & 1,75 & 0,60 & 1,00 & 1,67 & 3,33 & 17 \\
\hline \multicolumn{2}{|c|}{ Total } & 1,77 & 0,54 & 1,00 & 1,67 & 3,33 & 99 \\
\hline
\end{tabular}

El porcentaje «de acuerdo» y «muy de acuerdo», es muy elevado, superando en la mayoría el $90 \%$, no advirtiéndose diferencias significativas entre padres, tutores y orientadores en ninguna de las preguntas. 
- CENTROS EDUCATIVOS. Este grupo de variables recoge la siguiente información:

\begin{tabular}{|c|}
\hline \multicolumn{1}{|c|}{ Variables agrupadas } \\
\hline $\begin{array}{l}\text { 1- Proyectos y programas } \\
\text { específicos }\end{array}$ \\
\hline $\begin{array}{l}\text { 2- Centros públicos con } \\
\text { programas más adaptados }\end{array}$ \\
\hline $\begin{array}{l}\text { 3- Formación de los docentes } \\
\text { sobre altas capacidades }\end{array}$ \\
\hline $\begin{array}{l}\text { 4- Potenciación de la } \\
\text { identificación de los centros }\end{array}$ \\
\hline
\end{tabular}

\begin{tabular}{|c|c|c|c|c|c|c|c|}
\hline \multicolumn{7}{|c|}{ Centros Educativos } \\
\hline & & Media & d.t. & mín & $\mathbf{P ( 5 0 )}$ & Máx & N \\
\hline \multirow{3}{*}{ Relación } & $\mathrm{P} / \mathrm{M}$ & 2,99 & 0,50 & 2,00 & 2,86 & 3,71 & 37 \\
\cline { 2 - 8 } & $\mathrm{O}$ & 3,06 & 0,46 & 2,43 & 3,00 & 4,00 & 28 \\
\cline { 2 - 8 } & $\mathrm{T}$ & 2,57 & 0,34 & 2,00 & 2,57 & 3,14 & 17 \\
\hline \multicolumn{2}{|c|}{ Totales } & 2,95 & 0,48 & 2,00 & 2,86 & 4,00 & 42 \\
\hline
\end{tabular}

Se aprecia un descontento con «las ofertas» educativas que tiene este alumnado, ya que en general, todos se muestran disconformes con lo propuesto. No existen diferencias especialmente significativas entre padres, madres, orientadores y tutores, estando todos igualmente en desacuerdo (los tutores de forma más moderada).

Cuando se pregunta si existen suficientes programas especializados para este alumnado y de formación para los docentes, familias, tutores y orientadores están en desacuerdo con lo planteado en un porcentaje superior al $95 \%$.

- PROFESORADO. El módulo de información se obtuvo a partir de las siguientes variables:

\begin{tabular}{|l|}
\hline \multicolumn{1}{|c|}{ Variables agrupadas } \\
\hline $\begin{array}{l}\text { 1- Análisis de la situación de } \\
\text { aprendizaje }\end{array}$ \\
\hline $\begin{array}{l}\text { 2- Interés en informarse sobre las } \\
\text { altas capacidades }\end{array}$ \\
\hline 3- Coordinación \\
\hline $\begin{array}{l}\text { 4- Apoyo y comprensión al } \\
\text { alumnado }\end{array}$ \\
\hline
\end{tabular}

\begin{tabular}{|c|c|c|c|c|c|c|c|}
\hline \multicolumn{7}{|c|}{ Profesorado } \\
\hline \multirow{4}{*}{ Relación } & Media & d.t & Min & P(50) & max & N \\
\cline { 2 - 8 } & $\mathrm{P} / \mathrm{M}$ & 3,02 & 0,64 & 1,00 & 3,00 & 4,00 & 34 \\
\cline { 2 - 8 } & $\mathrm{O}$ & 2,90 & 0,60 & 1,00 & 2,80 & 4,00 & 31 \\
\cline { 2 - 8 } & $\mathrm{T}$ & 2,14 & 0,34 & 1,40 & 2,10 & 2,80 & 15 \\
\hline \multicolumn{2}{|c|}{ Totales } & 2,83 & 0,64 & 1,00 & 2,80 & 4,00 & 80 \\
\hline
\end{tabular}

Respecto a la actuación del profesorado, los tutores se muestran bastante de acuerdo con las afirmaciones que se plantean, mientras que padres y orientadores están más en desacuerdo. Estas diferencias son significativas $(\mathrm{p}=0.000)$ al considerar la variable promedio.

\section{Dimensiones o factores que caracterizan la situación}

Se ha realizado un análisis factorial, utilizado para «posicionar» unos grupos frente a otros (familias, tutores y orientadores), reemplazando los valores por la mediana.

Asimismo, para hacer un estudio de componentes principales sobre la matriz de correlaciones, se seleccionan inicialmente todos los autovalores mayores de uno (14 factores), aunque también se incluye, al estar muy próximo a uno (0.97), el autovalor 15. Finalmente, 
se consideran los 15 primeros factores, que nos revelan un 75,384\% de la variación total. Una vez etiquetadas las variables de puntuaciones factoriales, se utilizan para mirar «el posicionamiento» de los distintos grupos implicados en el análisis (padres, madres, tipo de colegio...). El método de rotación empleado es el de Normalización Varimax con Kaiser.

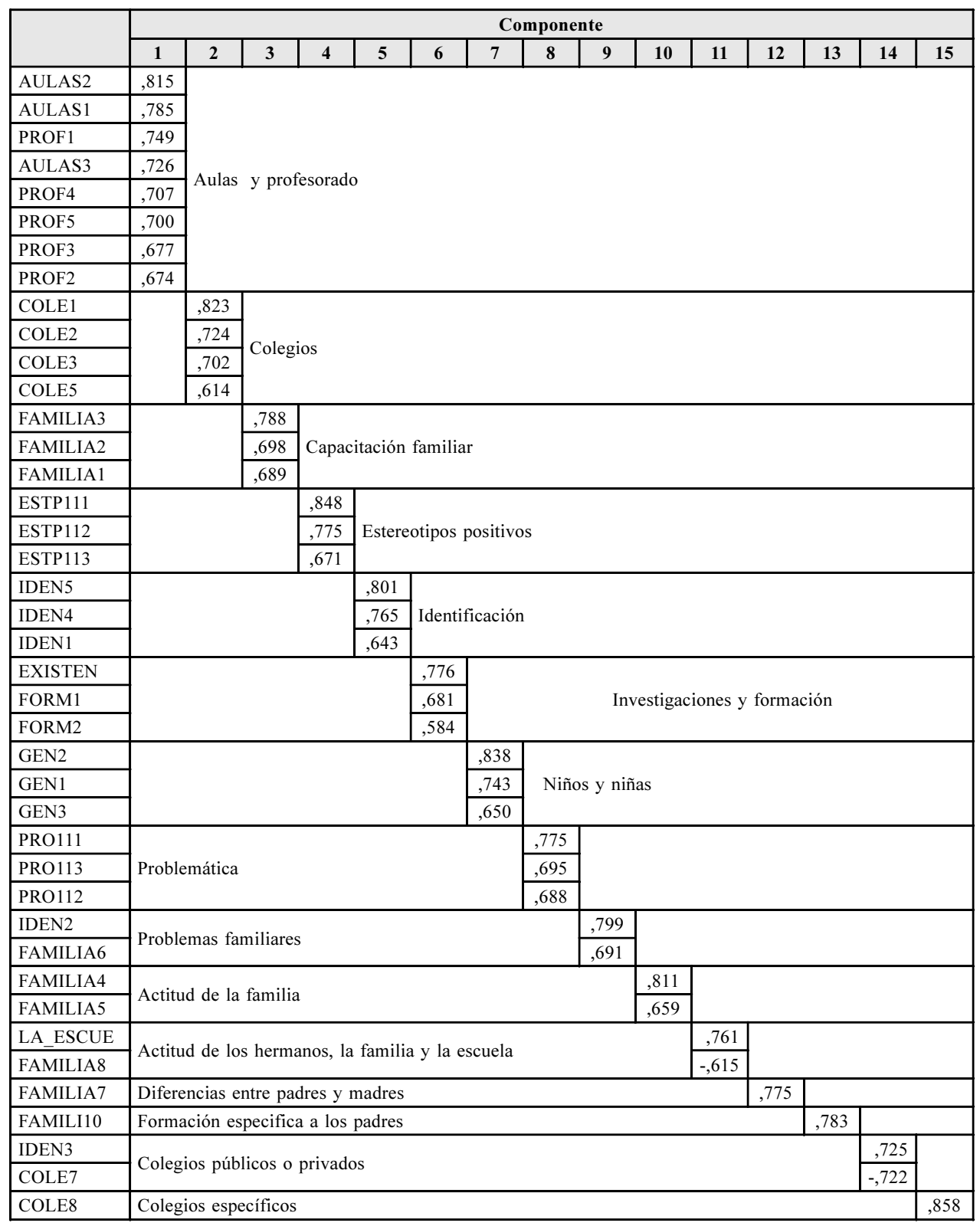


La primera columna recoge las variables del cuestionario abreviadas y la segunda incluye las puntuaciones obtenidas junto al nombre de la variable.

Como vemos, el primer factor está formado por ocho variables, referidas al tipo de enseñanza (aprendizaje autónomo, centrado en el alumnado, ambiente enriquecido) y la actitud del profesor (analiza la situación de aprendizaje, coordinación, formación, conocimiento y comprensión). Dada la homogeneidad de los contenidos, a este primer factor lo denominamos aulas y profesorado.

El segundo elemento colegios, recoge la actuación de los centros (atención a las necesidades de este alumnado, colaboración con la familia, elaboración de proyectos y programas educativos con referencia a estos alumnos, formación de los docentes en materia de superdotación). El tercer factor, capacitación familiar, se refiere a la angustia, la complejidad en la educación y la falta de preparación de las familias. En el cuarto se exponen los estereotipos positivos (suelen tener buenas notas, son brillantes y líderes, las familias tienen altas capacidades, les ayudan y estimulan constantemente). El siguiente elemento, identificación, agrupa las variables que indican la dificultad para detectar a este alumnado (minorías étnicas, ámbito rural y bajo rendimiento académico). Del mismo modo se va procediendo con el resto de los factores.

\section{Comportamiento diferencial}

Elaboradas las pruebas pertinentes de bondad de ajuste (Shapiro-Wilks, KolmogorovSmirnov con corrección de Lilliefors y Levene), podemos suponer que existe normalidad y homocedasticidad en las variables estudiadas.

Por último realizamos pruebas paramétricas para comprobar la influencia de los distintos factores. Concretamente, se realizan pruebas ANOVA de una vía (ver tabla 1).

Existen diferencias en relación con el factor colegios. Las familias que tienen hijas de altas capacidades se muestran más satisfechas con la actuación de los centros educativos, que las que tienen hijos. También se aprecian discrepancias en el factor niños y niñas, estando de nuevo «más de acuerdo» estas familias con la necesidad de que las niñas reciban más apoyo, debido a la dificultad de su identificación, ya que suelen ocultar su talento.

En las familias con hijos en programas de aceleración o de enriquecimiento, se advierten diferencias con respecto a aquellas cuyos hijos no realizan ningún programa específico. Estas disparidades se estiman: en el factor capacitación familiar (observan menos angustia y menos dificultad en su educación); en el factor investigaciones y formación (encuentran menos diferencias entre las políticas referidas al alumnado discapacitado y al de altas capacidades; están más satisfechas con la adecuación de los programas, la formación para el profesorado, el número de publicaciones y los equipos de investigación); y, por último, en el factor aulas y profesorado (están más de acuerdo con la actuación del profesorado respecto a la atención individualizada, la situación de aprendizaje, la coordinación y el interés del profesorado en la formación sobre altas capacidades).

También se observan diferencias entre las familias cuyos hijos realizan programas de aceleración y las que no llevan a cabo estos programas, respecto al factor aulas y profesora$d o$, (estando más satisfechas las primeras con el tipo de enseñanza y la actitud del profeso- 
TABLA 1.

\begin{tabular}{|c|c|c|c|c|c|c|}
\hline \multicolumn{2}{|l|}{ GÉNERO DEL NIÑO/A } & S.C. & GI & M.C. & $\mathbf{F}$ & Sig. \\
\hline \multirow{3}{*}{ Colegios } & Inter-grupos & 7,280 & 1 & 7,280 & 5,779 & 0,020 \\
\hline & Intra-grupos & 55,425 & 44 & 1,260 & & \\
\hline & Total & 62,705 & 45 & & & \\
\hline \multirow{3}{*}{ Niños y niñas } & Inter.-rupos & 4,706 & 1 & 4,706 & 5,565 & 0,023 \\
\hline & Intra-grupos & 37,211 & 44 & 0,846 & & \\
\hline & Total & 41,917 & 45 & & & \\
\hline \multicolumn{2}{|c|}{ INTERVENCIÓN EDUCATIVA } & S.C. & Gl & M.C. & $\mathbf{F}$ & Sig. \\
\hline \multirow{3}{*}{ Capacitación familiar } & Inter-grupos & 7,824 & 1 & 7,824 & 5,710 & 0,021 \\
\hline & Intra-grupos & 60,286 & 44 & 1,370 & & \\
\hline & Total & 68,110 & 45 & & & \\
\hline \multirow{3}{*}{$\begin{array}{l}\text { Investigaciones y } \\
\text { formación }\end{array}$} & Inter-rupos & 7,825 & 1 & 7,825 & 8,127 & 0,007 \\
\hline & Intra-rupos & 42,365 & 44 & 0,963 & & \\
\hline & Total & 50,189 & 45 & & & \\
\hline \multirow{3}{*}{ Aulas y profesorado } & Inter-rupos & 5,593 & 1 & 5,593 & 10,701 & 0,002 \\
\hline & Intra-rupos & 22,995 & 44 & 0,523 & & \\
\hline & Total & 28,587 & 45 & & & \\
\hline \multicolumn{2}{|c|}{ MODALIDAD DE CENTRO } & S.C. & GI & M.C. & $\mathbf{F}$ & Sig. \\
\hline \multirow{3}{*}{ Colegios } & Inter-grupos & 5,600 & 1 & 5,600 & 4,315 & 0,044 \\
\hline & Intra-grupos & 57,105 & 44 & 1,298 & & \\
\hline & Total & 62,705 & 45 & & & \\
\hline \multirow{3}{*}{ Estereotipos positivos } & Inter.gupos & 11,187 & 1 & 11,187 & 11,412 & 0,002 \\
\hline & Intra-grupos & 43,132 & 44 & 0,980 & & \\
\hline & Total & 54,319 & 45 & & & \\
\hline \multirow{3}{*}{$\begin{array}{l}\text { Actitud de los hermanos, } \\
\text { la familia y la escuela }\end{array}$} & Inter.gupos & 7,134 & 1 & 7,134 & 7,417 & 0,009 \\
\hline & Intra-grupos & 42,321 & 44 & 0,962 & & \\
\hline & Total & 49,455 & 45 & & & \\
\hline
\end{tabular}

rado) y al factor estereotipos positivos (creen más firmemente que suelen obtener mejores notas sin realizar mucho esfuerzo, son brillantes, etc.).

Nuevamente existen puntuaciones factoriales diferentes entre los padres y las madres que envían a sus hijos a centros concertados o públicos. Estas diferencias se concretan en los siguientes factores: colegios, estereotipos positivos, la actitud de los hermanos, la familia y la escuela.

\section{Conclusiones del trabajo}

Este trabajo recoge las conclusiones obtenidas tanto del análisis numérico como de la información aportada en las entrevistas personales. 


\section{Problemática emocional, social y escolar de los niños y niñas de altas capacidades}

A lo largo del trabajo se ha podido apreciar que, en general, todos los encuestados opinan que los niños de altas capacidades presentan desajustes sociales, se sienten aislados, diferentes y con problemas de relación, llegando, a veces, a padecer estrés, angustia, miedo, etc. Los aspectos que más predominan son la poca aceptación, atención y falta de apoyo.

Pocas familias creen en el buen desarrollo social, sin conflictos de sus hijos; y los orientadores, aunque están de acuerdo con esto, piensan que también existe diversidad y heterogeneidad.

Resulta difícil saber si familias y educadores están influenciados por estereotipos, pues en ocasiones, los docentes pueden considerar desajustes en el comportamiento de estos niños que en realidad no existen, como señalan Kolb y Jussim (1994) y Whitmore (1980). Por ello, una de las preocupaciones más importante en las familias es la modificación del comportamiento social de sus hijos (Gross, 1989). A pesar de esta creencia, una revisión con detenimiento de la bibliografía revela, que la mayoría de los estudiantes de altas capacidades se sienten muy bien con los de su edad, no existiendo ninguna problemática social (Robinson y Noble, 1991), lo que supone resultados contrarios a los del estudio.

Familias, orientadores y, en menor medida, tutores, son conscientes de que existe la posibilidad de una problemática escolar. Las familias centran este conflicto en la desmotivación y el aburrimiento, y piensan que sus hijos podrían conseguir sin esfuerzo un buen nivel académico. Tutores y orientadores argumentan que no es posible lograr un buen rendimiento académico sin esfuerzo.

Para Hallahan y Kaufman (1994) esta actitud familiar es consecuencia de estereotipos que impiden ver que a estos niños, generalmente, les gusta la escuela. A pesar de ello, García, Pérez y otros (1992) plantean que el porcentaje de fracaso escolar del alumnado de altas capacidades es semejante al del resto de los escolares (en torno al 30\%), confirmándose que no sólo no alcanzaban el nivel esperado para sus cualidades, sino que ni siquiera lograban llegar al nivel medio.

Csikszemtmihalyi, Rathunde, Whalen (1993) y Gardner (1993) coinciden en que la dedicación de los padres y, sobre todo, de las madres es fundamental para poder llegar a alcanzar unas metas, afirmación que, por otro lado, se contrapone con los resultados del estudio; puesto que las familias no se atribuyen un papel relevante. Sin embargo, docentes y educadores destacan la importancia del factor genético y de la aportación familiar.

\section{Género}

No hay diferencias de opinión entre los padres y las madres en ninguna de las cuestiones planteadas, pero sí se aprecian discrepancias cuando nos referimos al género de los hijos. Las familias, particularmente las que tienen hijas, y los orientadores (aunque con un alto porcentaje de desconocimiento) piensan que es dificil diagnosticar las altas capacidades a las niñas. Creen necesario un mayor apoyo y confianza en sus posibilidades, debido a la ambigüedad del papel de la mujer en la sociedad. 
Aunque se observa un gran desconocimiento sobre el tema, los docentes no advierten diferencias en cuanto al género en la identificación de altas capacidades. De igual modo no consideran que las niñas necesiten más apoyo.

En muchas ocasiones, como sostiene Benito (1993), se han identificado altas capacidades de manera casual, ya que los padres y las madres acudían en busca de apoyo para su hijo superdotado y la alta capacidad de la hija pasaba desapercibida. Tal como plantea Faludi (1991), muchos conflictos sobre el papel de la mujer aún perviven en nuestra sociedad y, cómo no, en la educación; desde la familia y las escuelas se envían mensajes contradictorios sobre la realización académica femenina, lo que impide, según Leroux (1992), que muchas niñas manifiesten su talento.

Coriat (1990) opina, que estas actitudes son debidas a que la sociedad presiona a la mujer a no ser intelectualmente superior al hombre si no quiere sentirse socialmente marginada. Lo primordial es que hogar y escuela aúnen sus esfuerzos para conseguir que estas niñas acepten sus aptitudes, Bell (1990), Ellis y Willinsky (1997).

Tanto los padres como las madres con niñas de altas capacidades, mantienen menos expectativas que las familias con hijos de estas características, sin embargo, el grado de satisfacción con la educación recibida en los centros, es superior. De este modo se confirman las conclusiones de otros trabajos (Fox y Turner, 1981), en los que se demostraba, como las familias depositan mayores esperanzas en sus hijos.

\section{Educación de hijos e hijas}

Las familias, se consideran víctimas de la incomprensión social, y esto les produce frustración, angustia y preocupación, aunque suelen reconocer que con el tiempo este sentimiento disminuye. Los problemas no surgen en el seno familiar, sino que son consecuencia de la actitud de los demás. Esta angustia es descrita en muchas investigaciones, como las de Colangelo (1997) y Keirouz (1990).

Los orientadores captan mejor la ansiedad en las familias que los propios implicados, sin embargo, los docentes sostienen que, en general, no existe una problemática especial, llegando incluso a pensar que se suelen sentir felices con esta situación.

Todos consideran que la falta de preparación y la complejidad que conlleva educar a estos niños, determina la necesidad de que las familias reciban orientación, apoyo psicológico y formación, para poder atender eficazmente las necesidades de sus hijos.

Resulta muy importante que en tales circunstancias los padres y las madres dispongan de una ayuda para poder mantener el necesario equilibrio afectivo e intelectual. En este sentido, Domínguez y Pérez (1998) ponen de manifiesto que lo que se precisa es orientación y apoyo para liberarse de mitos y estereotipos, con el fin de poder comprender y apoyar a su hijo/a.

Padres y madres confiesan que su mayor preocupación es la integración social de su hijo o hija, en cambio, tutores y orientadores aseguran que anteponen los éxitos escolares al desarrollo emocional y social de los niños. No obstante, un número considerable de orientadores cree que la tónica dominante es la diversidad, y que todo depende de la educación familiar. 
En general, coinciden en que no se suelen producir celos entre los niños de altas capacidades y sus hermanos, aunque algunas familias y orientadores centran la problemática familiar cuando tienen hermanos; afirman que sí se producen cambios y surgen roces en la convivencia. Tener niños con estas características puede complicar las relaciones entre hermanos según Weissler y Landau (1992). Cornell (1984) en su estudio llegó a la conclusión de que los hermanos «menos aptos» pueden verse afectados negativamente en su autoestima, ajuste emocional, competitividad y cooperación.

\section{Centros Educativos}

Toda la comunidad educativa está en desacuerdo con la educación que se oferta a los niños de altas capacidades, poniendo de manifiesto que no se les tiene en cuenta en los proyectos y programas $y$, que no hay una política clara de identificación en los colegios. Parece confirmarse que las demandas de los padres y las madres no son irracionales, sino lógicas y justas (George, 1988). Frente a estos resultados, tenemos los estudios de Arocas, Martínez y Regadera (1999), quienes plantean que las familias sienten satisfacción con la atención y educación que reciben sus hijos en los centros.

Se han encontrado diferencias significativas en las opiniones de las familias cuyos hijos participan en programas de aceleración y enriquecimiento. Así pues, padres y madres cuyos hijos realizan algún programa, piensan que las familias sufren menos tensión, angustia, están más preparadas y que no es tan complicado educar a estos niños de forma adecuada. Creen que este alumnado genera menos problemas de los que, en un principio cabría esperar, y están profundamente convencidos de la necesidad de más programas, investigaciones y publicaciones. De igual modo, se sienten menos insatisfechos con las actividades realizadas en el aula, con la actitud y preparación del profesorado.

Todos los encuestados reconocen su desconocimiento acerca de qué tipo de centro es el más adecuado para el alumnado de altas capacidades, pero coinciden en que los centros específicos no son los más convenientes para su desarrollo integral.

Se aprecia, en general, más satisfacción, con la atención educativa y la colaboración familia-centro, en los padres y las madres que envían a sus hijos a colegios concertados; estando más convencidas de la buena relación entre los hermanos y de la mayor eficacia de los centros específicos. Consideran que el alumnado de altas capacidades necesita esforzarse para conseguir los objetivos si quieren desarrollar las altas capacidades. Todo parece apuntar que su concepción sobre la educación les lleva a exigir más rendimiento y esfuerzo a sus hijos y esto, a su vez, genera más satisfacción al comprobar que van alcanzando mejores resultados.

Algunos docentes piensan que para formarse precisan más horas libres, años sabáticos $e$ incentivos. Hay sugerencias que van desde la mejora de las tutorías a recibir clases extraescolares.

Los orientadores destacan que en las aulas, a veces, sólo se realizan adaptaciones de objetivos y, eso si el tutor se quiere implicar y colabora, por lo que muchos solicitan materiales de identificación y enseñanzas más personalizadas, con especial incidencia en la diversidad. 


\section{Profesorado}

Padres y madres coinciden en la necesidad de cambiar el comportamiento del profesorado, desterrando actitudes impropias tales como pasividad, desinterés e incluso, hostilidad encubierta. Por otra parte, las familias parecen olvidar lo importante que es ser positivo, paciente y perseverante en su comunicación con la escuela (Acedera, 2000).

Se solicita más formación, recursos humanos y materiales; orientadores y padres la piden tanto para sí mismas como para toda la Comunidad educativa, los docentes la demandan para ellos. Así pues, se muestran convencidos de que esa formación les va a ayudar no sólo para conocer las características y los métodos más adecuados para tratar a este alumnado, sino también para su correcta identificación.

Frente a la opinión de las familias y los orientadores que piensan que en las aulas no se están realizando actividades que favorezcan el aprendizaje de este alumnado, los tutores como parte implicada, creen que el profesorado analiza la situación de aprendizaje, trabaja coordinadamente y muestra una actitud comprensiva hacia el alumnado de altas capacidades.

\section{Valoraciones complementarias}

Dada su extensión, otros análisis realizados sobre el cuestionario no han sido expuestos en sus apartados correspondientes, aunque consideramos interesante resaltar las conclusiones obtenidas:

\section{La identificación}

Las familias y los orientadores señalan que las dificultades en la identificación no son debidas a las características familiares, a no ser que se pertenezca a alguna minoría étnica. La mayoría coincide en que un bajo rendimiento escolar dificulta la identificación de este alumnado. Según Dowdall, Colangelo (1982) y Renzulli (1978), sólo se considera real la superdotación cuando se manifiesta con resultados elevados, atribuidos a los individuos de rendimiento académico alto.

Los docentes relacionan altas capacidades con rendimiento académico y reconocen abiertamente la necesidad de recibir mayor formación, no sólo para poder trabajar adecuadamente en las aulas sino para poder identificar a este alumnado. A pesar de ello, son más reacios que las familias y los orientadores a reconocer las deficiencias que tienen los centros en la identificación.

Padres y madres opinan que la Administración debe diseñar estrategias y cambios para mejorar los métodos de identificación, no dejando la situación a la voluntad de los docentes.

\section{La política educativa}

La necesidad de información de familias y docentes, respecto a la legislación, queda de manifiesto al reconocer su alto grado de desconocimiento. Los orientadores piensan que las 
leyes, por muchas razones, no se ponen en práctica. Toda la Comunidad educativa es partidaria de que la Administración debería actuar clara e inequívocamente en este tema, dedicando más medios y recursos.

Todos señalan que se otorga diferente tratamiento a este alumnado en comparación con el que presenta discapacidad; siendo escasos los equipos de investigación y las publicaciones periódicas sobre superdotación.

Se constata que los programas son insuficientes y que el profesorado no tiene una adecuada formación. Los orientadores piden la aplicación de programas específicos, atendiendo sobre todo a los aspectos sociales, familiares y afectivos.

\section{Colaboración familia-escuela}

Las relaciones entre los centros y los padres con hijos de altas capacidades no son demasiado positivas.

Las pocas familias que mantienen una buena comunicación con el centro escolar, y creen que la actuación de éste es adecuada, lo atribuyen a la suerte, a que sus exigencias son mínimas o a que su actitud es muy paciente y comprensiva.

Se confirma que las diferencias entre los padres y los centros son habituales, tal como destaca Butler-Por, 1993; incluso en este estudio, en donde casi todo el alumnado presenta buen rendimiento académico.

Muchas familias, pese a mantener una actitud crítica y negativa con los centros e incluso confesar abiertamente que las relaciones con los docentes no son buenas, reconocen que en numerosas ocasiones no colaboran ni dedican el tiempo suficiente para mejorar la situación. Buscan soluciones y piden más información y contacto con el profesorado. Aseguran que es necesaria la implicación de todo el centro, demandan mayor colaboración y un cambio en todos los sentidos.

Los docentes también se muestran preocupados, manifestando la necesidad de recibir formación y de que se impliquen los centros, aunque, por otro lado, se quejan de las desproporcionadas exigencias de las familias, consecuencia de las grandes expectativas que tienen para sus hijos. Winner (1996), sin embargo, afirma que a la mayoría se les aconseja no presionar demasiado a sus hijos ya que se podrían convertir en personas resentidas, desengañadas y depresivas, que acabarían por perder todo el interés por el conocimiento. Esto conlleva bajas expectativas para este alumnado, conformándose con unos niveles muy modestos de conocimientos.

Todos consideran necesario mantener una estrecha comunicación entre la familia y la escuela. Proponen una relación tutorial seria y planificada con más coordinación y seguimiento.

\section{Resumen}

Este estudio refleja muchas coincidencias entre los tres grupos de personas entrevistadas. Tanto familias como orientadores y tutores, señalan que el estilo de vida cambia cuando se confirma que su hijo o hija posee altas capacidades. Son conscientes de la posibilidad 
de que estos niños tengan problemas emocionales, sociales o escolares, de la falta de preparación y de la necesidad de orientación que tienen los padres y las madres. Están de acuerdo en que la actuación de la Administración y de los colegios no es la más adecuada, y de que es preciso realizar más investigaciones y desarrollar programas educativos adaptados a este alumnado. Las críticas de las familias a los centros, coinciden con las de tutores y orientadores, aunque a veces, solamente con las de los orientadores (siendo éstos, en algunas ocasiones, más exigentes con la actuación escolar que las propias familias). A pesar de esto, tutores y orientadores opinan que los padres y las madres esperan demasiado de sus hijos, les preocupa más los éxitos escolares y se crean expectativas muy elevadas que normalmente no se cumplen, por no estar, ni los centros, ni los docentes, suficientemente preparados. Este hecho provoca que las familias se sientan defraudadas y resentidas, a pesar de los intentos de los docentes por mejorar las relaciones.

El principal problema consiste en conseguir satisfacer las exigencias que las familias demandan, ya que solicitan, principalmente, una atención individualizada y unos programas educativos específicos. El profesorado carece de una adecuada formación y del apoyo necesario tanto de la Administración como de los centros educativos y, a veces incluso de las familias, para poder atender esas demandas. A pesar de ello, consideran las reivindicaciones familiares lógicas y justas. Parece pues necesario abandonar esa actitud expectante y, a menudo, indecisa de los responsables de la educación y potenciar un verdadero cambio que permita cumplir con todos los compromisos y desafíos.

Se observa, no obstante, mayor satisfacción en los progenitores que tienen a sus hijos realizando programas de aceleración o enriquecimiento, ya que presentan menos problemas en su entorno. Se muestran, asimismo, con más seguridad y confianza respecto a la actuación de los colegios y del profesorado. Sin embargo, los padres y las madres que envían a sus hijos a los centros públicos sienten menor satisfacción, ya que creen que éstos no suelen responder a sus expectativas.

Por último, se pone de manifiesto la necesidad de ampliar y continuar las investigaciones en este campo.

\section{Puntos de encuentro}

\section{Familias, Orientadores y Docentes}

1. Existencia de una posible problemática escolar (desmotivación y aburrimiento), social (problemas de relación así como poca aceptación), y emocional (desarrollo asíncrono) del alumnado de altas capacidades.

2. Gran desconocimiento sobre la situación actual de las niñas de altas capacidades.

3. El alumnado de altas capacidades con bajo rendimiento no suele ser identificado.

4. No se potencia la identificación de las altas capacidades en los centros.

5. Reconocimiento de una situación familiar más compleja cuando los hijos tienen altas capacidades.

6. Necesidad de apoyo, información y orientación familiar.

7. Escasos e insuficientes programas educativos para este alumnado. 
8. Necesidad de incrementar las investigaciones y publicaciones sobre el tema.

9. Más apoyo y atención al alumnado con discapacidad.

10. Atención inadecuada por parte de los centros educativos.

11. Deficiente formación de los docentes sobre las altas capacidades.

12. Desconocimiento sobre el tipo de centro más adecuado: público o privado.

13. Los centros específicos no son los más convenientes para el desarrollo integral de estos niños.

14. Falla la comunicación y la relación familia-centro educativo.

15. Es preciso un compromiso real entre la familia y los colegios, con más información, contactos, coordinación y planificación.

\section{Docentes y Orientadores}

1. Los niños de altas capacidades necesitan esforzarse para conseguir alcanzar las metas educativas.

2. Existen antecedentes genéticos en las altas capacidades.

3. Las familias suelen apoyar, ayudar y estimular.

4. Las familias están más interesadas en los éxitos escolares que en la situación personal y social.

5. Los padres y las madres no silencian las altas capacidades de sus hijos.

\section{Familias y docentes}

1. Las leyes sobre el alumnado de altas capacidades son inadecuadas.

\section{Familias y Orientadores}

1. Las niñas se identifican peor que los niños.

2. Las familias sienten angustia y preocupación cuando conocen que su hijo o hija es de altas capacidades.

3. Falla la atención individualizada, la coordinación y el apoyo en los centros.

4. No se realizan las actividades adecuadas para este alumnado en las aulas.

\section{Referencias bibliográficas}

Acedera, A. y Sastre, S. (1998). La superdotación. Madrid: Síntesis.

Acedera, A. (2000). Niños superdotados. Madrid: Pirámide.

Arocas, E., Martínez, P. y Regadera, A. (1998). Actitudes y expectativas de las familias respecto a la institución. En Congreso Internacional para alumnos superdotados y talentosos. Zaragoza, 8-11 de Julio. 
Ballering, L. y Koch, A. (1983). Family relations when a child is gifted. Gifted Child Quarterly, 28(3), 140-143.

Bell, L. A. (1990). The gifted woman as impostor. Advanced Development. A Journal on Adult Giftedness, 2(1), 85-94.

Benito, Y. (1993). Características más comunes a los niños superdotados y necesidades educativas especiales. Evaluación de las funciones integradoras metacognitivas y de potencial de aprendizaje en niños superdotados. En Comunicaciones I Congreso Internacional de Psicología y Evaluación. Madrid 1991: Editorial Complutense.

Bloom, B. (1985). Developing talent in young people. New York: Ballantine Books.

Bridges, S. (1973). IQ-150. Londres: Priory Press.

Butler-Por, N. (1993). Underachieving Gifted Students. En K. A. Heller, F. J. Monks y A. H. Passow (Eds.), International handbook of research and development of giftedness and talent, (pp. 649668). Oxford: Pergamon Press.

Colangelo, N. y Dettman, D. (1980). A functional model for counseling parents of gifted students. Gifted Child Quarterly, 31(2), 59-64.

Colangelo, N. y Dettman, D. (1983). A review of research on parents and families of gifted children. Exceptional Children, 50(1), 20-27.

Colangelo, N. y Davis, G. A. (1991). Handbook of gifted education. Boston: Allyn and Bacon.

Coleman, L. J. (1985). Schooling the gifted. Menlo Park, CA. Addison-Wesley Publishing Co.

Coriat, A. (1990). Los niños superdotados. Barcelona: Herder.

Cornell, D. G. (1983). Gifted Children: The impact of positive labeling on the family system. American Journal of Orthopsychiatry, 53, 322-336.

Cornell, D. G. (1984). Families of gifted children. Michigan: UMI Research Press

Csikszentmihalyi, M., Rathunde, K. y Walen, S. (1993). Talented teenagers: The roots of success and failure. New York: Cambridge University Press.

Dirks, J. (1979). Parent's reactions to identification of the gifted. Roeper Review, 2(2), 9-10.

Domínguez Rodríguez, P. y Pérez Sánchez, L. (1998). Actitudes de la Sociedad y la Familia ante los adolescentes superdotados. En Intervención Psicológica en la adolescencia. VIII Congreso INFAD. Pamplona.

Dowdall, C. y Colangelo, N. (1982). Underace undeclared war against American women. New York: Crown.

Fox, L. y Turner, L. (1981). Gifted and creative females. The iddle school years. American Middle School Education, 4, 17-23.

Gallagher, R. (1991). Programs for gifted students in Chicago public schools: yesterday, today and tomorrow. Gifted Child Today, 14(6), 16-27.

García-Alcañiz, E., Pérez-Sánchez, L. y otros. (1992). Diferencias intelectuales y de rendimiento académico entre chicos-chicas bien dotados y de la media. I Congreso Internacional de Psicología y Educación. Madrid.

Gardner, H. (1993a). Creating minds: An Anatomy of creativity seen through the lives of Freud, Einstein, Picasso, Stravinsky, Eliot, Graham and Gandhi. New York: BasicBooks.

Gardner, H. (1995). Mentes Creativas. Barcelona: Paidós Testimonio.

George, R. (1988). Tracking and ability grouping. Middle School Journal, 20(1), 21-28.

Gottfried, A.W., Gottfried, A.E., Bathurst, K. y Guerin, D.W. (1994). Gifted IQ: Early developmental aspects. The Fullterton longitudinal study. New York: Plenum. 
Gross, M. U. M. (1989). The pursuit of excellence or the search for intimacy? The forced-choice dilemma of gifted youth. Roeper Review, 11(4), 189-194.

Hackney, H. (1981). The gifted child, the family, and the school. Gifted Child Quarterly, 25(2), 51-54.

Hallahan, D. y Kauffman, J. M. (1994). Exceptional children. Introduction to special education. Boston: Allyn and Bacon.

Hill, J. (1980). The family. En toward adolescence: The middle school years. Seventy-ninth yearbook of the study of education. Chicago: University of Chicago Press.

Howe M. J. (1990). The origins of exceptional abilities. Oxford: Basil Blackwell.

Keirouz, K. S. (1990). Concerns of Parents of Gifted Children. A Research Review. Gifted Child Quarterly, 34(2), 56-63.

Kolb, K. y Jussim, L. (1994). Teacher expectations and underachieving gifted children. Roeper Review, 17, 26-30.

Leroux, J. A. (1992). Reaching beyond the barriers: A follow-up study of gifted females. En F. Monks y W. Peters, (Eds.), (pp. 162-170).

Martínez, M y Castiglione, F. (1995). Romper estereotipos sobre la superdotación. Ponencia presentada en el II Congreso Internacional de Psicología y Pedagogía. Madrid, 16-18 de Noviembre de 1995.

Martínez, M y Castiglione, F. (1996). Las familias con hijos e hijas de altas capacidades. Orientación e intervención en la familia del niño superdotado. Curso M.E.C. Madrid, Marzo de 1996.

Mathews, F. N., West, J. D. y Hosie, T. W. (1986). Understanding families of academically gifted children, Roeper Review, 9, 40-42.

Miller, A (1991). El drama del niño bien dotado. Barcelona: Tusquets.

Moon, S., Kelly, K. y Feldhusen, J. (1997). Specialized counseling services for gifted youth and their families: A needs assessment. Gifted Child Quarterly, 41(1), 16-25.

Ness, B. (1988). Establishing programs for parents of the gifted child. Gifted Child Today, 11(1), 2426.

Pérez L. (1993). Diez palabras clave sobre superdotados. Navarra: Verbo Divino.

Pérez, L. (1995). El asesoramiento y la orientación vocacional de los alumnos superdotados. En F. Rivas, Manual de asesoramiento y orientación vocacional. Madrid: Síntesis.

Pressey, S. (1955). Conceding the nature and nurture of genius. Scientific Monthly, 81, 123-129.

Renzulli, J. (1978). What makes giftedness? Reexamining a definition, Phi Delta Kappan, 60, 180184 y 261.

Robinson, N. M. y Noble, K. D. (1991). Social-emotional development and adjustment of gifted children. En M. C. Wang, M. C. Reynolds y H. J. Walberg (Eds.), Handbook of special education: Research and practice, Vol.4.Emerging programs (pp.57-76). New York: Pergamon Press.

Ross, A. O. (1964). The exceptional child in the family. New York: Grune y Stratton.

Selltiz, C. (1976). Métodos de investigación en las relaciones sociales. Madrid: Rialp.

Silverman, L. K. (1986b). What happens to the gifted girl? En C. J. Maker (Ed.), Critical issues in gifted education, Vol. 1: Defensible programs for the gifted (pp. 43-89). Austin: TX. Pro-Ed.

Tabackman, M. (1976). A study of family psycho-social environment and its relationship to academic achievement in gifted adolescents. Illinois: Urbana-Champaign.

Verhaären, P.R. (1991). Educación de los alumnos superdotados. Madrid: MEC.

Wallace, B. (1988). La educación de los niños más capaces. Madrid: Visor. 
Wallace, T. y Walberg, H. (1987). Personality traits and childhood environments of eminent essayists. Gifted Child Quarterly, 31(32), 65-69.

Weissler, K. y Landau, E. (1992). Characteristics of families with no, one or more than one gifted child. Journal of Psychology, 127(2), 143-152.

Whitmore, J. (1980). Giftedness, conflict and underachievement. Boston: Allyn and Bacon.

Winner, E. (1996). Gifted Children: Myths and realities. New York: Basic Books.

Fecha de recepción: 18-VII-01

Fecha de revisión: 10-IX-01

Fecha de aceptación: 16-X-01 